\title{
Association de betteraves fourragères à du foin pour des vaches laitières
}

\author{
JP Dulphy ${ }^{1}$, J Rouel 2 \\ ${ }^{1}$ INRA, station de recherches sur la nutrition des herbivores, 63122 St-Genès-Champanelle; \\ 2 INRA, domaine d'Orcival, 63120 Rochefort-Montagne, France
}

(Reçu le 4 septembre 1990; accepté le 25 avril 1991)

\begin{abstract}
Résumé - Dans un essai réalisé avec 24 vaches laitières en milieu de lactation, l'effet d'un apport de $3,1 \mathrm{~kg}$ de MS de betteraves fourragères à $17 \%$ de MS a été étudié avec une ration de base constituée de foin de bonne qualité. Les animaux ont ingéré en moyenne $12 \mathrm{~kg}$ de MS de foin plus $6,1 \mathrm{~kg}$ de betteraves + concentrés, pour une production de lait journalière de $21,4 \mathrm{~kg}$. Le taux de substitution des betteraves au foin a été élevé puisqu'il a été de 0,9 , correspondant à une valeur d'encombrement des betteraves de 0,96 UEL/kg de MS. La valeur énergétique estimée des betteraves a été au moins égale à celle retenue dans les tables d'alimentation de l'INRA (1988), soit 1 UFL $k g$ de MS. En présence de betteraves, le taux protéique du lait produit a augmenté, mais non significativement, de 0,75 point. Par contre le taux butyreux n'a pas été modifié.
\end{abstract}

betterave fourragère / foin / vache laltlère / ingestion / valeur nutritlve

Summary - Association of fodder beet with hay in dairy cow feeding. The effect of supplementing a basal diet based on good quality hay with $3.1 \mathrm{~kg}$ of fodder beet DM (at $17 \%$ DM) was tested in 24 dairy cows in mid-lactation. The animals ingested on an average $12 \mathrm{~kg}$ of hay dry matter and $6.1 \mathrm{~kg}$ of beets plus concentrates for a daily milk production of $21.4 \mathrm{~kg}$. The substitution rate of beet for hay was high (0.9) corresponding to a forage fill value of 0.96 LFU/kg of DM. The energy value of beets was at least equal to that given in the INRA feed tables (1988), ie 1 UFL/kg of DM. The use of fodder beet increased the protein content of milk, but not significantly, by 0.75 point. It did not alter the fat content.

fodder beet / hay / dairy cow / feed intake / nutritional value 


\section{INTRODUCTION}

Compte tenu de leur valeur énergétique élevée, les betteraves fourragères peuvent être très utiles pour améliorer la valeur des rations à base de foin. Cette association a été très recommandée avant le développement de l'ensilage d'herbe. Cette recommandation était aussi basée sur le fait que l'apport de betteraves, dont la valeur d'encombrement était comparable à celle d'un aliment concentré (Vérité et al, 1974), permettait d'augmenter le niveau d'ingestion de la ration. De Brabander et al (1974 et 1976) ont d'ailleurs largement confirmé l'observation précédente.

Les betteraves présentent aussi généralement l'avantage d'améliorer la composition du lait (Rémond, 1985) et de favoriser la repise de poids vif des animaux (Vérité et Journet, 1973).

Les conditions d'utilisation des betteraves ayant fortement évolué surtout à cause de l'augmentation du potentiel de production des vaches laitières, il nous est apparu utile, comme avec les ensilages d'herbe (Dulphy et al, 1990), de réactualiser les résultats concernant l'association de betteraves à du foin. Un dernier essai a donc été entrepris sur le Domaine INRA d'Orcival avec les mêmes objectifs que précédemment, à savoir :

- vérifier la valeur d'encombrement et la valeur énergétique des betteraves dans une ration à base de foin pour des vaches bonnes productrices;

- confirmer l'effet positif de l'apport de betteraves sur les taux protéique et butyreux du lait.

En outre, comme dans les essais avec ensilage d'herbe, les animaux ont reçu des betteraves dès le vêlage pour étudier la façon dont évoluait la production de lait en début de lactation.

\section{MATÉRIEL ET MÉTHODES}

Pour cette étude, qui a comporté 2 parties, 24 vaches laitières multipares de race Pie Noire, placées en stabulation entravée sans paille, ont été utilisées au cours de l'hiver 1989-1990.

Dans une première partie, tous les animaux ont été conduits de la même façon pour la période allant de 2 semaines avant leur vêlage (octobre-début novembre) à un minimum de 12 semaines de lactation. Ils ont alors reçu chaque jour : $4 \mathrm{~kg}$ de MS de betteraves non hachées; 2,7 kg de MS de foin de deuxième cycle; du foin de premier cycle à volonté; un concentré d'équilibre pour couvrir leurs besoins (Hoden et al, 1988). Son apport a augmenté à partir du vêlage pour atteindre en moyenne $8,6 \mathrm{~kg}$ de MS au cours de la quatrième semaine de lactation; $1 \mathrm{~kg}$ de tourteau de soja supplémentaire pendant les 6 premières semaines de lactation.

Lorsque tous les animaux ont atteint leur douzième semaine de lactation (environ $17^{\circ}$ pour les premiers vêlés), ils ont été répartis en 3 lots comparables (numéro de lactation, stade de lactation, poids vif, production de lait, composition du lait, quantités ingérées). Les valeurs retenues ont été celles des 2 semaines précédant la mise en lot qui a eu lieu fin janvier. Une semaine a ensuite été consacrée à passer les animaux sur leurs régimes respectifs.

Dans une seconde partie, de 12 semaines également, les animaux ont reçu chaque jour :

- lot 1 : foin de premier cycle à volonté, $4 \mathrm{~kg}$ de foin de deuxième cycle, des concentrés (orge + tourteau de soja) en vue de couvrir les besoins (UFL et PDI);

- lot 2 : même régime que pour le lot 1 avec un apport de $3 \mathrm{~kg}$ de MS de betteraves en supplément afin de calculer un taux de substitution, mais en effectuant des apports iso-PDI par rapport au lot 1;

- lot 3 : même régime que pour le lot 2 , mais avec une réduction de l'apport de concentré de 2,3 UFL. L'objectif était alors d'effectuer des apports d'énergie théoriquement identiques à ceux du lot 1.

Pendant cette période, les apports de concentré ont été calculés pour couvrir chaque semaine les besoins attendus des animaux, en tenant compte d'une diminution théorique de la 
production de lait de $2 \%$ par semaine. Ces concentrés, ainsi que les betteraves, ont été distribués en 2 fois par jour. Les animaux ont reçu aussi un aliment minéral vitaminisé pour couvrir leurs besoins ( $200 \mathrm{~g} / \mathrm{j}$ ).

Les caractéristiques des aliments sont données dans le tableau I. Ce sont les mêmes foins qui ont été utilisés durant les 2 parties de l'étude. Leur valeur était bonne. Les betteraves contenaient à peine plus de cendres que celles des tables INRA (1988). Leur teneur moyenne en matière sèche a été de $17,3 \%$.

Les quantités ingérées ont été déterminêes individuellement après pesée des quantités offertes et refusées, tous les jours pour les aliments offerts en quantité limitée et $4 \mathrm{j}$ par semaine pour le foin de premier cycle. Les quantités de lait ont été pesées tous les j et leur composition a été déterminée 2 j par semaine. Les animaux ont été pesés toutes les 2- 4 semaines.

Les résultats ont été ensuite analysés par un programme d'analyse de variance (SAS) et les moyennes ajustées en fonction des résultats des 2 semaines précédant la mise en lot utilisés comme covariables.

Tableau I. Caractéristiques des aliments utilisés.

\section{RÉSULTATS}

\section{Début de lactation}

Les résultats moyens concernant les 12 semaines du début de la lactation sont présentés au tableau II. Pendant cette période, les animaux ont produit $30 \mathrm{~kg}$ de lait et perdu $20 \mathrm{~kg}$ de poids vif corrigé des variations de contenu digestif (équivalence de $1 \mathrm{~kg}$ de MS de foin pour $4,5 \mathrm{~kg}$ de contenu et de $1 \mathrm{~kg}$ de MS de concentré pour $2,25 \mathrm{~kg}$ de contenu).

Compte tenu des interactions digestives estimées (Faverdin et al, 1987) et des pertes de poids vif, la valorisation énergétique de la ration a été bonne (tableau III). Cependant, par comparaison aux animaux ayant reçu de l'ensilage d'herbe sans betteraves dans les essais précédents (Dulphy et al, 1990), la production de lait a atteint rapidement son maximum (en $3 e$

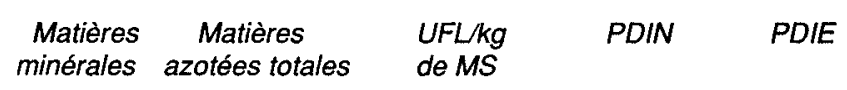

$g / k g$ de $M S$

$g / k g$ de MS

$\begin{array}{llrrrr}\text { Foin de } 1^{\mathrm{er}} \text { cycle } & 81 & 117 & 0.74 & 73 & 79 \\ \text { Foin de } 2^{\circ} \text { cycle } & 90 & 169 & 0.82 & 106 & 95 \\ \text { Betteraves } & 60 & 65 & 1,00^{*} & 38 & 85 \\ \text { Tourteau de soja } & 77 & 476 & 1,14^{*} & 348^{\star} & 241^{\star} \\ \text { Orge } & 30 & 115 & 1,16^{\star} & 79^{\star} & 102^{\star} \\ \text { Concentré d'équilibre } & 85 & 186 & 1,05^{\star} & 115^{\star} & 115^{\star}\end{array}$

" D'après les tables INRA (1988). 
Tableau II. Principaux résultats expérimentaux (valeurs ajustées en milieu de lactation).

\begin{tabular}{|c|c|c|c|c|c|}
\hline Effectifs（） & $\begin{array}{c}\text { Début } \\
\text { de lactation } \\
\text { (24) }\end{array}$ & $\begin{array}{c}1 \\
(8)\end{array}$ & $\begin{array}{c}\text { Milieu de la } \\
2 \\
(8)\end{array}$ & $\begin{array}{r}\text { ion. Lots: } \\
3 \\
(8)\end{array}$ & ETR \\
\hline \multicolumn{6}{|l|}{$\begin{array}{l}\text { Quantités ingérées } \\
\text { (kg de MS) }\end{array}$} \\
\hline foin $1^{\text {er }}$ cycle & 6,8 & $9,66 a$ & $7,13 b$ & $8,13 c$ & 0,67 \\
\hline foin $2^{\theta}$ cycle & 2,7 & 3,8 & 3,7 & 3,6 & \\
\hline betteraves & 4,0 & 0 & 3,06 & 3,06 & \\
\hline concentré équilibré & 6,8 & - & - & - & \\
\hline tourteau de soja & 0,43 & 0,87 & 0,43 & 0,87 & \\
\hline orge & - & 3,90 & 4,30 & 1,90 & \\
\hline total & 20,8 & 18,23 & 18,62 & 17,56 & \\
\hline \multicolumn{6}{|l|}{ Production } \\
\hline lait brut (kg) & 30,0 & 20,55 & 21,04 & 20,56 & 1,45 \\
\hline lait à $4 \%$ de MG $(\mathrm{kg})$ & 30,3 & 21,28 & 21,77 & 21,15 & 1,40 \\
\hline matières grasses $(\mathrm{g})$ & 1218 & 871 & 894 & 860 & 60 \\
\hline matières protéiques $(\mathrm{g})$ & 957 & 662 & 695 & 671 & 41 \\
\hline \multicolumn{6}{|l|}{ Composition du lait } \\
\hline taux buryreux (\%o) & 40,6 & 42,4 & 43,1 & 42,0 & 2,1 \\
\hline taux protéique $(\%)$ & 31,9 & 32,4 & 33,2 & 33,1 & 1,0 \\
\hline cellules $(1000 / \mathrm{ml})$ & - & 353 & 267 & 303 & 138 \\
\hline Poids vif $(\mathrm{kg})$ & 637 & 642 & 644 & 633 & 11 \\
\hline Variation du $\mathrm{PV}(\mathrm{g} / \mathrm{j})$ & -234 & +202 & +156 & +203 & 151 \\
\hline
\end{tabular}

Seules les valeurs suivies des lettres $a, b, c$ sont significativement differentes.

semaine pour le lait à $4 \%$ de matière grasse; en $4^{\mathrm{e}}$ semaine pour le lait brut) (fig 1).

\section{Milieu de lactation}

Tous traitements confondus, les animaux ont ingéré en moyenne $18,1 \mathrm{~kg}$ de $\mathrm{MS} / \mathrm{j}$ pour une production laitière de $21,4 \mathrm{~kg}$ à 4\% de MG (tableau II).

Entre le lot 1 et le lot 2, l'apport de betteraves a fait baisser la quantité de foin in- gérée de $2,63 \mathrm{~kg}$ de MS, soit un taux de substitution foin/betteraves de 0,86 . Entre les lots 3 et 2, le taux de substitution du foin au concentré a été égal à 0,56 .

La capacité d'ingestion moyenne des vaches utilisées est estimée à 17 UEL (21 $\mathrm{kg}$ de lait; $640 \mathrm{~kg}$ de poids vif - Faverdin et al, 1987).

En attribuant au foin de deuxième cycle une valeur de 1 UEL/kg de MS et en tenant compte du taux de substitution entre le foin de premier cycle et le concentré de 0,56 , la valeur de ce foin est estimée à 
Tableau II. Apports et bilans nutritifs journaliers moyens pour les 12 semaines d'essai (sur résultats non ajustés).

\begin{tabular}{|c|c|c|c|c|}
\hline & \multirow[t]{2}{*}{$\begin{array}{c}\text { Début } \\
\text { de laction }\end{array}$} & \multicolumn{3}{|c|}{$\begin{array}{l}\text { Milieu de lactation } \\
\text { Lots }\end{array}$} \\
\hline & & 1 & 2 & 3 \\
\hline Apports & 18,88 & 15,78 & 16,87 & 14,89 \\
\hline Interactions $\mid$ (en UFL/J) & 2,23 & 0,66 & 1,39 & 0,90 \\
\hline $\begin{array}{l}\text { Bilan énergétique } \\
\text { (apports - besoins) }\end{array}$ & $-1,08$ & $-0,28$ & $-0,38$ & $-1,19$ \\
\hline besoins énergétiques & \multirow[b]{2}{*}{1,06} & \multirow[b]{2}{*}{1,02} & \multirow[b]{2}{*}{1,02} & \multirow[b]{2}{*}{1,08} \\
\hline Rapport & & & & \\
\hline Apports PDI limitants $(g / j)$ & 1866 & 1573 & 1520 & 1511 \\
\hline Bilans en PDI (g/j) & -2 & +150 & +27 & +105 \\
\hline
\end{tabular}

1,07 UEL/kg de MS et la valeur théorique du concentré à 0,60 UEL/kg de MS $(1,07 \times$ $0,56)$. En reportant enfin toutes ces valeurs pour les lots 2 et 3 , la valeur d'encombrement des betteraves atteint respectivement 0,92 et 1,00 , soit en moyenne $0,96 \mathrm{UEL} / \mathrm{kg}$ de MS.

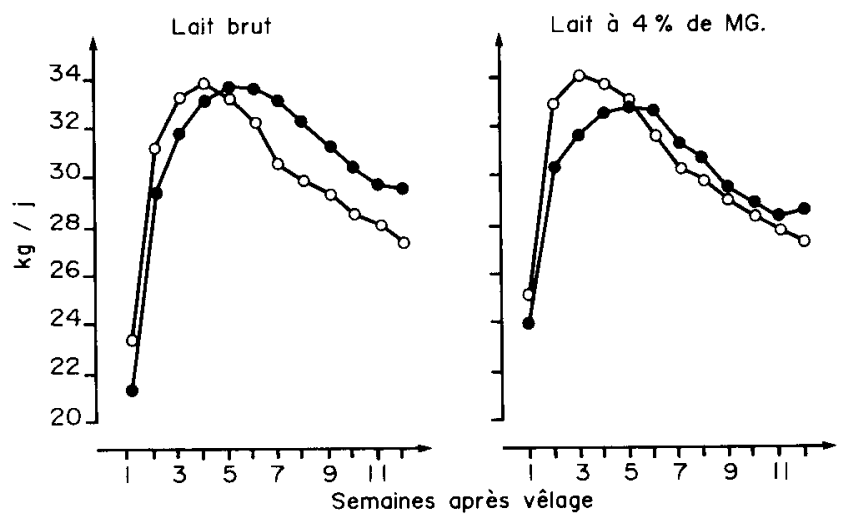

Fig 1. Comparaison des productions laitières en début de lactation : $\longrightarrow$ : lots ensilage d'herbe témoins (Dulphy et al, 1990) ( $n=18$ vaches); $0-0$ : lot foin + betteraves $(n=24$ vaches).
Les quantités de lait produites ont été comparables entre les 3 lots. Les taux butyreux n'ont pas non plus été modifiés significativement par l'apport de betteraves. II en est de même pour les taux protéiques, malgré une augmentation observée de $0,75 \mathrm{~g} / \mathrm{kg}$ suite à l'apport de betteraves. 
Dans ces conditions, seule la production de protéines par animal et par jour a augmenté légèrement avec l'apport de betteraves, mais de façon non significative.

Les autres paramètres enregistrés n'ont pas été non plus significativement différents : gains de poids et nombre de cellules par $\mathrm{ml}$ de lait, malgré une tendance à la baisse pour les cellules en présence de betteraves.

Les bilans énergétiques figurent dans le tableau III. Ils tiennent compte des variations de poids vif (Faverdin et al, 1987) et sont nettement négatifs en début de lactation et pour le lot 3 en milieu de lactation. Ils sont pratiquement équilibrés pour les lots 1 et 2 en milieu de lactation. Compte tenu des performances obtenues, il apparaît donc que la valorisation de la ration 3 est excellente $(+6 \%$ par rapport à la ration témoin). La valorisation de la ration 2 a été identique à celle de la ration 1 . Notons enfin qu'il n'y a pas eu de déficit en PDI pour les différentes rations étudiées.

\section{DISCUSSION ET CONCLUSION}

La première partie de cette étude ne correspond pas à un essai, mais à une simple observation. Compte tenu du nombre d'animaux utilisés, cette observation est cependant relativement fiable, même si elle n'est pas généralisable. Le démarrage rapide de la production de lait observée est dû très probablement à un potentiel supérieur des animaux utilisés ici par rapport à ceux des essais avec ensilage d'herbe (Dulphy et al, 1990). La forme de la courbe de lactation montre que les animaux ont été davantage sous-alimentés avec le foin, malgré l'apport de betteraves, qu'avec l'ensilage d'herbe, même si les bilans énergétiques moyens sont comparables $(-1.08$ avec le foin contre -1.30 avec l'ensilage). II aurait donc fallu apporter plus de concentré pour avoir un démarrage plus classique de la lactation.

Dans la seconde partie de l'étude, la valeur moyenne d'encombrement des betteraves a été de $0,96 \mathrm{UEL} / \mathrm{kg}$ de $\mathrm{MS}$, ce qui correspond à un taux de substitution de 0,90 (1,07 UEL/kg de MS de foin / 0,96 UEL/kg de MS de betteraves). Les valeurs trouvées dans la littérature sont très variables : 0,5 pour vérité et al (1974); 0,40 et 0,46 pour De Brabander et al (1974); 0,65 et 0,70 pour De Brabander et al (1976); 0,75 pour Jans (1983). La valeur que nous trouvons est donc élevée, ce qui est peut-être dû au fait que les besoins énergétiques des animaux dans les lots 2 et 3 étaient correctement couverts, ce qui peut augmenter le taux de substitution (Dulphy et al, 1987).

Dans cette étude, par contre, l'utilisation énergétique des betteraves a été tout à fait correcte, puisque en prenant la valeur de 1 UFL/kg de MS (pour un apport supérieur à $3 \mathrm{~kg}$ de MS - INRA, 1988), le rapport besoins/apports-interactions a été supérieur à 1. Cela laisse d'ailleurs supposer que les betteraves ont eu une valeur énergétique supérieure à 1 UFL/kg de MS, probablement à cause de leur valeur d'encombrement élevée (Dulphy et al, 1990).

Enfin, même si la différence n'est pas significative, il y a eu une légère augmentation du taux protéique du lait, augmentation de $0,75 \mathrm{~g} / \mathrm{kg}$, comparable à celle de 0,85 trouvée précédemment par Dulphy et al (1990).

Pour conclure, ce travail confirme la valeur énergétique élevée des betteraves fourragères, tout en laissant quelques incertitudes quant à leur valeur d'encombrement. L'emploi de cet aliment pourra néanmoins être envisagé comme celui d'un aliment concentré, plutôt que comme un composant de la ration de base. 


\section{REMERCIEMENTS}

Nous tenons à remercier l'ADBFM (Association pour le développement de la betterave fourragère) de son concours qui a permis la réalisation de cette étude.

\section{RÉFÉRENCES}

De Brabander DL, Aerts JV, Boucque $\mathrm{CH}$, Buysse FX, Moermans RJ (1974) Influence spécifique des betteraves fourragères sur l'ingestion chez les vaches laitières. Rev Agric 6, 1479-1491

De Brabander DL, Aerts JV, Boucque $\mathrm{CH}$, Buysse FX (1976) Influence spécifique de betteraves fourragères sur l'ingestion chez les vaches laitières (II). Rev Agric 3, 593606

Dulphy JP, Faverdin P, Micol D, Bocquier F (1987) Révision du système des unités d'encombrement. Bull Tech CRVZ Theix INRA 70, 35-48

Dulphy JP, Rouel J, Bony J (1990) Association de betteraves fourragères à de l'ensilage d'herbe pour des vaches laitières. INRA Prod Anim 3 (3), 195-200

Faverdin P, Hoden A, Coulon JB (1987) Recommandations alimentaires pour les vaches laitières. Bull Tech CRVZ Theix INRA 70, 133152

Hoden A, Coulon JB, Faverdin P (1988) Alimentation des vaches laitieres. In : INRA 1988, 135-158

INRA (1988) Alimentation des Bovins, Ovins ot Caprins (R Jarrige, ed) Ed INRA Publ, $471 \mathrm{p}$

Jans (1983) Importance des betteraves fourragères dans la ration des vaches laitières. In: La tachetée rouge du Simmental $n^{\circ} 6,50-54$

Rémond $B$ (1985) Influence de l'alimentation sur la composition du lait de vache. 2. Taux protéique : facteurs généraux. Bull Tech CRZV Theix INRA 62, 53-68

Vérité R, Hoden A, Journet M (1974) Distribution de betteraves en quantités élevées à des vaches laitières. Bull Tech CRZV Theix INRA 15-20

Vérité $R$, Journet $M$ (1973) Utilisation de quantités élevées de betteraves par les vaches laitières : étude de l'ingestion, de la digestion et des effets sur la production. Ann Zootech 22, 219-235 